12 Morris A, Wachter RM, Luce J et al. Improved survival with highly active antiretroviral therapy in HIV-infected patients with severe Pneumocystis carinii pneumonia. AIDS 2003; 17: 73-80.

J Antimicrob Chemother 2017

doi:10.1093/jac/dkw580

Advance Access publication 25 January 2017

\section{Pneumocystis jirovecii pneumonia: still a concern in patients with haematological malignancies and stem cell transplant recipients- authors' response}

\author{
Catherine Cordonnier ${ }^{1 *}$, Alexandre Alanio ${ }^{2}$, \\ Simone Cesaro ${ }^{3}$, Georg Maschmeyer ${ }^{4}$, \\ Hermann Einsele ${ }^{5}$, J. Peter Donnelly ${ }^{6}$, \\ Philippe M. Hauser ${ }^{7}$, Katrien Lagrou ${ }^{8}$, \\ Willem J. G. Melchers ${ }^{9}$, Jannik Helweg-Larsen ${ }^{10}$, \\ Olga Matos ${ }^{11}$, Stéphane Bretagne ${ }^{2}$ and Johan \\ Maertens ${ }^{12}$ on behalf of the Fifth European Conference \\ on Infections in Leukemia (ECIL-5 ${ }^{\dagger)}$, a joint venture of \\ The European Group for Blood and Marrow \\ Transplantation (EBMT), The European Organization for \\ Research and Treatment of Cancer (EORTC), the \\ Immunocompromised Host Society (ICHS) and The \\ European LeukemiaNet (ELN)
}

${ }^{1}$ Department of Haematology, Henri Mondor Teaching Hospital, Assistance Publique-hôpitaux de Paris, and Université Paris-EstCréteil, Créteil, France; ${ }^{2}$ Parasitology-Mycology Laboratory, Groupe Hospitalier Lariboisière Saint-Louis Fernand Widal, Assistance Publique-Hôpitaux de Paris (AP-HP), Université ParisDiderot, Sorbonne Paris Cité, and Institut Pasteur, Unité de Mycologie Moléculaire, CNRS URA3012, Centre National de Référence Mycoses Invasives et Antifongiques, Paris, France; ${ }^{3}$ Department of Haematology, Oncoematologia Pediatrica, Policlinico G. B. Rossi, Verona, Italy; ${ }^{4}$ Department of Haematology, Oncology and Palliative Care, Ernst-von-Bergmann Klinikum, Potsdam, Germany; ${ }^{5}$ Department of Internal Medicine II, Julius Maximilians University, Würzburg, Germany; ${ }^{6}$ Department of Haematology Radboud University Medical Center, Nijmegen, The Netherlands; ${ }^{7}$ Institute of Microbiology, Lausanne University Hospital and University, Lausanne, Switzerland; ${ }^{8}$ Department of Microbiology and Immunology, KU Leuven - University of Leuven, Leuven, Belgium and National Reference Center for Mycosis, Department of Laboratory
Medicine, University Hospitals Leuven, Leuven, Belgium; ${ }^{9}$ Department of Medical Microbiology, Radboud University Medical Center, Nijmegen, The Netherlands; ${ }^{10}$ Department of Infectious Diseases, Rigshospitalet-Copenhagen University Hospital, Copenhagen, Denmark; ${ }^{11}$ Medical Parasitology Unit, Group of Opportunistic Protozoa/HIV and Other Protozoa, Global Health and Tropical Medicine, Instituto de Higiene e Medicina Tropical, Universidade Nova de Lisboa, Lisboa, Portugal;

${ }^{12}$ Department of Haematology, Acute Leukaemia and Stem Cell Transplantation Unit, University Hospitals Leuven, Campus Gasthuisberg, Leuven, Belgium

*Corresponding author. Haematology Department, Henri Mondor University Hospital, 51 Avenue du Maréchal de Lattre de Tassigny, 94000 Créteil, France. Tel: +331498120 59; Fax: +331 498120 67; E-mail: catherine.cordonnier@aphp.fr

†ECIL-5 participants are listed in the Acknowledgements section.

Sir,

We would like to thank Antinori et al. ${ }^{1}$ for their comments on the European Conference on Infections in Leukemia (ECIL) guidelines for the management of Pneumocystis jirovecii pneumonia (PCP). ${ }^{2}$

Antinori et al. ${ }^{1}$ listed four points, which we would like to address one by one.

\section{Mode of acquisition of PCP in haematology patients}

The mode of acquisition of PCP in haematology patients is uncertain, but there is no reason to think that it is different from that in other immunocompromised patients. Exposure to $P$. jirovecii through inhalation begins early in life, as shown by autopsy series, and PCR and serological screenings. ${ }^{3-6}$ The high rate of mixtures of genotypes during PCP (up to 90\%) also supports continuous exposure to P. jirovecii from the environment via other humans. ${ }^{7}$ When investigating outbreaks in solid organ transplant units using genotyping, only some of the cases were shown to be due to inter-human or environmental transmission. ${ }^{8,9}$ To our knowledge, only two outbreaks have been reported in haematology wards. The first one included five cases in 6 months in the same haematology unit, but without any molecular investigation. ${ }^{10}$ The second study established a genetic link using internal transcribed spacer (ITS) sequencing between the strains for two out of eight haematology patients (and two out of six HIV-positive patients), suggesting that person-toperson transmission was relatively infrequent. ${ }^{11}$ Recently, transmission between patients from different wards was reported with the help of six new short tandem repeat markers located in the nuclear genome. ${ }^{12}$ However, even with this highly discriminant genotyping method, we could not differentiate between reactivation and a new infection. ${ }^{12}$ Despite the lack of solid data reflected by a grading of only C-III, ECIL proposed that patients in haematology should, nonetheless, avoid contact with those infected with PCP. ${ }^{13}$

\section{Differences in clinical presentation and hypoxaemia between patients with and without HIV infection}

Antorini et al. ${ }^{1}$ show that two-thirds of their patients with HIV infection had a $\mathrm{PaO}_{2}<70 \mathrm{mmHg}$. However, the $\mathrm{PaO}_{2}$ of patients with PCP, but without HIV infection, was not presented. This makes any conclusion about $\mathrm{PaO}_{2}$ at diagnosis difficult. We agree with Antorini et al. ${ }^{1}$ that $\mathrm{PaO}_{2}$ at presentation can be 
highly dependent on the quality of local care, especially rapid access to bronchoalveolar lavage. Indeed, the delay from the onset of symptoms to establishing a diagnosis of PCP was variable according to the year of diagnosis and the centres involved and may well have had an impact on the severity at presentation. ${ }^{14-19}$ Nevertheless, apart from open studies reporting high rates of hypoxaemia in patients with PCP and underlying haematological conditions, parallel comparisons of those with and without HIV infection showed that the degree of respiratory failure at presentation (often before admission to the ICU), whether assessed by $\mathrm{PaO}_{2}, \mathrm{SatO}_{2}$ or $\mathrm{PaO}_{2} / \mathrm{FiO}_{2}$, was significantly higher in those without HIV infection ${ }^{15-17,20}$ and the need for ventilation was significantly more frequent in three series, ${ }^{14,17,19}$ but not in a fourth one. ${ }^{18} \mathrm{~A}$ recent French series, which was not available at the ECIL-5 meeting, confirms this pattern since only 25 (11\%) of 223 patients with AIDS required invasive mechanical ventilation, compared with 98 (30.5\%) of 321 with another underlying condition, including 111 with haematological diseases. ${ }^{19}$ Moreover, a diagnosis of AIDS was associated with lower mortality, whereas the converse was true for receipt of an allogenic HSCT and the need for mechanical ventilation. ${ }^{19}$

\section{Lactate dehydrogenase (LDH) levels at presentation}

LDH levels at diagnosis were $39 \%-46 \%$ higher among patients with HIV infection than among those without. ${ }^{14,18,21}$ However, the ECIL-5 laboratory diagnosis group concluded that this discussion had been superseded by the ready availability of more specific laboratory tools, such as PCR or $\beta$-D-glucan detection, making further mention of LDH elevation for the diagnosis of PCP redundant. ${ }^{22}$ We consider, however, that an unexplained serum LDH elevation in the presence of a suggestive clinical presentation could be taken into account when deciding upon initiation of PCP treatment, providing quick, direct investigations. ${ }^{23}$

\section{Mortality rates in HIV-positive patients}

The mortality due to PCP is usually assessed at day 90 after onset. In series of patients with HIV infection reported from 2000 s, it was reported to vary from $9.6 \%{ }^{17}$ to $30 \% .{ }^{18,20}$ By contrast, a recent French survey reported an in-hospital mortality rate of $4 \%$ for those with AIDS, which was significantly lower than the $27 \%$ rate found for those with other underlying conditions. $^{19}$

Besides the higher mortality, PCP in those not infected with HIV tends to occur very abruptly and carries a high risk of ICU transfer. Hence, the haematology community should be alerted to this possibility and needs to ensure adequate and timely diagnostic and prevention strategies are developed and implemented to offer the best outcomes.

\section{Acknowledgements}

\section{ECIL-5 meeting participants}

Samir Agrawal, UK; Murat Akova, Turkey; Alexandre Alanio, France; Mahmoud Aljurf, Saudi Arabia; Dina Averbuch, Israel; Thomas Berg, Germany; Ola Blennow, Sweden; Stéphane Bretagne, France; Roger Brüggemann, the Netherlands; Thierry Calandra, Switzerland; Elio Castagnola, Italy; Simone Cesaro, Italy; Catherine Cordonnier, France; Oliver Cornely, Germany; Rafael De La Camara, Spain; Peter Donnelly, the Netherlands; Lubos Drgona, Slovakia; Rafael Duarte, Spain; Hermann Einsele, Germany; Dan Engelhard, Israel; Corrado Girmenia, Italy; Ruth Hargreaves (Gilead Sciences), UK; Philippe Hauser, Switzerland; Jannick Helweg-Larsen, Denmark; Raoul Herbrecht, France; Hans Hirsch, Switzerland; Petr Hubacek, Czech Republic; Christopher Kibbler, UK; Galina Klyasova, Russia; Michal Kouba, Czech Republic; Bart-Jan Kullberg, the Netherlands; Katrien Lagrou, Belgium; Per Ljungman, Sweden; Johan Maertens, Belgium; Vincent Mallet, France; Oscar Marchetti, Switzerland; Georg Maschmeyer, Germany; Olga Matos, Portugal; Willem Melchers, the Netherlands; Malgorzata Mikulska, Italy; Patricia Munoz, Spain; Christina Orasch, Switzerland; Livio Pagano, Italy; Antonio Pagliuca, UK; Olaf Penack, Germany; George Pettrikos, Greece; Zdenek Racil, Czech Republic; Patricia Ribaud, France; Valérie Rizzi-Puechal (Pfizer), France; Emmanuel Roilides, Greece; Janos Sinko, Hungary; Anna Skiada, Greece; Monica Slavin, Australia; Jan Styczynski, Poland; Frederic Tissot, Switzerland; Lorraine Tweddle (Astellas), UK; Florian van Boemmel, Germany; Marie von Lilienfeld-Toal, Germany; Claudio Viscoli, Italy; Katherine Ward, UK; Craig Wood (MSD), USA.

\section{Transparency declarations}

S. B. received a consultant honorarium from Myconostica. All the remaining authors have none to declare.

\section{Author contributions}

All authors have approved the content of the manuscript. C. C. and A. A. drafted this letter and all authors approved the final version.

\section{References}

1 Antinori S, Binda F, van den Bogaart L et al. Comment on: Pneumocystis jirovecii pneumonia: still a concern in patients with haematological malignancies and stem cell transplant recipients. J Antimicrob Chemother 2017; 72: 1264-66.

2 Cordonnier C, Cesaro S, Maschmeyer G et al. Pneumocystis jirovecii pneumonia: still a concern in patients with haematological malignancies and stem cell transplant recipients. J Antimicrob Chemother 2016; 71: 2379-85.

3 Gajdusek DC. Pneumocystis carinii; etiologic agent of interstitial plasma cell pneumonia of premature and young infants. Pediatrics 1957; 19: 543-65.

4 Ponce CA, Gallo M, Bustamante R et al. Pneumocystis colonization is highly prevalent in the autopsied lungs of the general population. Clin Infect Dis 2010; 50: 347-53.

5 Totet A, Meliani L, Lacube P et al. Immunocompetent infants as a human reservoir for Pneumocystis jirovecii: rapid screening by non-invasive sampling and real-time PCR at the mitochondrial large subunit rRNA gene. J Eukaryotic Microbiol 2003; 50 Suppl: 668-9.

6 Vargas SL, Hughes WT, Santolaya ME et al. Search for primary infection by Pneumocystis carinii in a cohort of normal, healthy infants. Clin Infect Dis 2001; 32: 855-61.

7 Alanio A, Gits-Muselli M, Mercier-Delarue S et al. Diversity of Pneumocystis jirovecii during infection revealed by ultra-deep pyrosequencing. Front Microbiol 2016; 7: 733.

8 de Boer MG, de Fijter JW, Kroon FP. Outbreaks and clustering of Pneumocystis pneumonia in kidney transplant recipients: a systematic review. Med Mycol 2011; 49: 673-80.

9 Yiannakis EP, Boswell TC. Systematic review of outbreaks of Pneumocystis jirovecii pneumonia: evidence that $P$. jirovecii is a transmissible organism and the implications for healthcare infection control. J Hosp Infect 2016; 93: 1-8.

10 Ong YL, Jones FG. A cluster of suspected Pneumocystis carinii pneumonia following intensive chemotherapy in a Belfast haematology unit. Ulster Med J 1998; 67: 104-9.

11 Helweg-Larsen J, Tsolaki AG, Miller RF et al. Clusters of Pneumocystis carinii pneumonia: analysis of person-to-person transmission by genotyping. QJM 1998; 91: 813-20.

12 Gits-Muselli M, Peraldi MN, de Castro N et al. New short tandem repeatbased molecular typing method for Pneumocystis jirovecii reveals 
intrahospital transmission between patients from different wards. PLoS One 2015; 10: e0125763.

13 Maertens J, Cesaro S, Maschmeyer G et al. ECIL guidelines for preventing Pneumocystis jirovecii pneumonia in patients with haematological malignancies and stem cell transplant recipients. J Antimicrob Chemother 2016; 71 : 2397-404.

14 Ewig S, Bauer T, Schneider C et al. Clinical characteristics and outcome of Pneumocystis carinii pneumonia in HIV-infected and otherwise immunosuppressed patients. Eur Respir J 1995; 8: 1548-53.

15 Kovacs J, Hiemenz J, Macher A et al. Pneumocystis carinii pneumonia: a comparison between patients with the acquired immunodeficiency syndrome and patients with other immunodeficiencies. Ann Intern Med 1984; 100: 663-71.

16 Limper A, Offord K, Smith T et al. Pneumocystis carinii pneumonia. Differences in lung parasite number and inflammation in patients with and without AIDS. Am Rev Respir Dis 1989; 140: 1204-9.

17 Mansharamani N, Garland R, Delaney D et al. Management and outcome patterns for adult Pneumocystis carinii pneumonia, 1985 to 1995. Comparison of HIV-associated cases to other immunocompromised states. Chest 2000; 118: 704-11.
18 McKinnell JA, Cannella AP, Kunz DF et al. Pneumocystis pneumonia in hospitalized patients: a detailed examination of symptoms, management, and outcomes in human immunodeficiency virus (HIV)-infected and HIVuninfected persons. Transpl Infect Dis 2012; 14: 510-8.

19 Roux A, Canet E, Valade $S$ et al. Pneumocystis jirovecii pneumonia in patients with or without AIDS, France. Emerging Infect Dis 2014; 20: 1490-7.

20 Monnet X, Vidal-Petiot E, Osman D et al. Critical care management and outcome of severe Pneumocystis pneumonia in patients with and without HIV infection. Crit Care 2008; 12: R28.

21 Nuesch R, Bellini C, Zimmerli W. Pneumocystis carinii pneumonia in human immunodeficiency virus (HIV)-positive and HIV-negative immunocompromised patients. Clin Infect Dis 1999; 29: 1519-23.

22 Alanio A, Hauser PM, Lagrou K et al. ECIL guidelines for the diagnosis of Pneumocystis jirovecii pneumonia in patients with haematological malignancies and stem cell transplant recipients. J Antimicrob Chemother 2016; 71: 2386-96.

23 Maschmeyer G, Helweg-Larsen J, Pagano L et al. ECIL guidelines for treatment of Pneumocystis jirovecii pneumonia in non-HIV-infected haematology patients. J Antimicrob Chemother 2016; 71: 2405-13. 